\title{
Risk prediction of early decline in renal function in diabetic kidney disease with algorithm including fractional excretion of glycated amino acids \\ Naila Rabban ${ }^{1}$ Alberto de a Fuente, ${ }^{2}$ and Paul J Thornalley ${ }^{2}$
}

\author{
${ }^{1}$ Department of Basic Medical Science, College of Medicine, QU Health, Qatar University, Doha, Qatar. and
}

\section{Diabetes Research Center, Qatar Biomedical Research Institute, Hamad Bin Khalifa University, Qatar Foundation, Doha, Qatar}

BACKGROUND: Diabetic kidney disease occurs in ca. $40 \%$ patients with diabetes. Approximately 1 in 5 patients with type 1 diabetes mellitus (T1DM) and 1 in 3 patients with type 2 diabetes mellitus (T2DM) develop early decline in renal function (EDRF), requiring renal dialysis after 5 - 20 years. Currently, at the time of normoalbuminuria (NA) or new onset microalbuminuria (MA, incipient diabetic nephropathy), it is uncertain which patients are at risk of EDRF (Persson \& Rossing, Kidney Internat Suppl 8, 2-7, 2018)

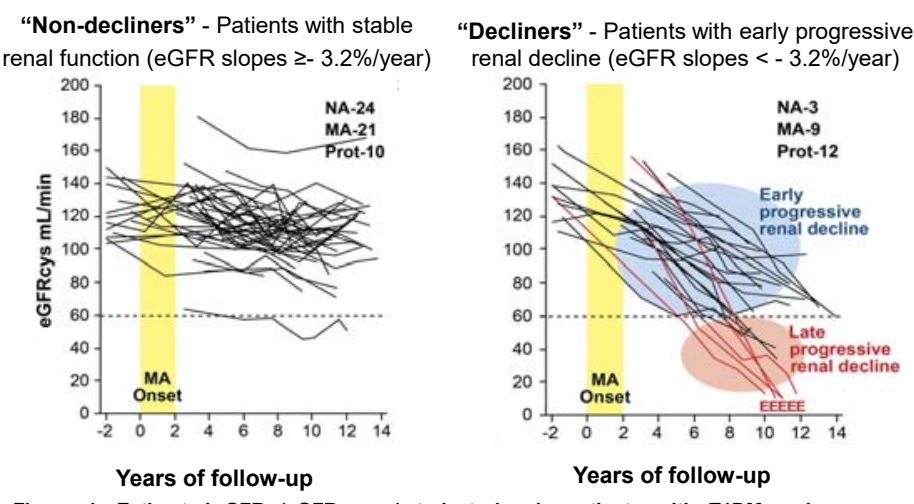

Figure 1. Estimated GFR (eGFRcr-cys) trajectories in patients with T1DM and normoalbuminuria (NA) and progressive renal decline (loss $\geq 3.3 \%$ per year) during 4-10 years of follow-up. Lines in red indicate presence of macroalbuminuria. E, ESRD. From: Krolewski et al., Diabetes Care 37, 226-234, 2014

With Joslin Kidney Study investigators, we found patients with T1DM who later developed EDRF (Decliners) have higher fractional excretion (FE) of 6 glycated amino acids - fructosyl-lysine and 5 advanced glycation endproducts (AGEs), compared to patients with stable renal function (Non-decliners) (Rabbani et al., Scientific Reports 10, 12709, 2020). However, FEs of single glycated amino acids could not classify Decliners or Non-decliners.

AIM: The aim of this study was to apply artificial intelligence machine learning to develop diagnostic algorithms to classify Decliners and Nondecliners by optimal combination of levels of glycated and oxidized amino acids in plasma and urine, related FEs and conventional clinical chemistry variables.

RESULTS: Algorithm training and testing with FE of glycation free adducts gain an algorithm providing strong, often conclusive evidence for identification of patients with T1DM at future risk of EDRF.
Optimum algorithm features:

$\mathrm{A} 1 \mathrm{C}, \log [\mathrm{ACR}], \mathrm{FE}_{\mathrm{CMA}}, \mathrm{FE}_{\mathrm{G}-\mathrm{H} 1}$, and

$[\mathrm{CML}]_{\text {Plasma }}$

Classification performance:

Accuracy $=87 \% \pm 4 \%$

- Sensitivity $=74 \% \pm 9 \%$

Specificity $=91 \% \pm 4 \%$

AUROC $=0.90$

- Positive likelihood ratio $\mathrm{LR}+=11.0$, Key indicating strong, often conclusive risk prediction of EDRF
Receiver operating characteristic (ROC) curve

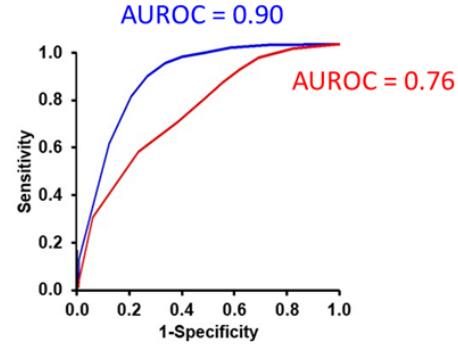

Algorithm with A1C \& logACR and $[\mathrm{CML}]_{\text {plasma }}$ Algorithm with $A 1 C$ \& logACR $F E_{C M A}, F_{G-H 1}$

Abbreviations: CMA, $\mathrm{N}_{\omega}$-carboxymethylarginine; $\mathrm{CML}, \mathrm{N}_{\varepsilon}$-carboxymethyl-lysine; and $\mathrm{G}-\mathrm{H} 1$, glyoxal-derived hydroimidazolone; and $\mathrm{CML}$
PATIENTS AND METHODS: Patients with T1DM with stable renal function ( $n=63$ ) and EDRF ( $n=22$ ) were recruited for this study. Data on levels of 14 glycated and oxidized amino acids in plasma, urine, related FEs, glycated hemoglobin $\mathrm{A} 1 \mathrm{C}, \log$ (urinary albumin creatinine ratio, ACR), age, gender and duration of diabetes at the time of new onset microalbuminuria were included as features in algorithm development (Rabbani et al., Scientific Reports 10, 12709, 2020). Algorithms were trained and tested on $90 \% / 10 \%$ data split, repeated 1000 times, using the Extreme Gradient Boosting method.

\section{"Extreme gradient boosting" xgboost https://xgboost.ai/}
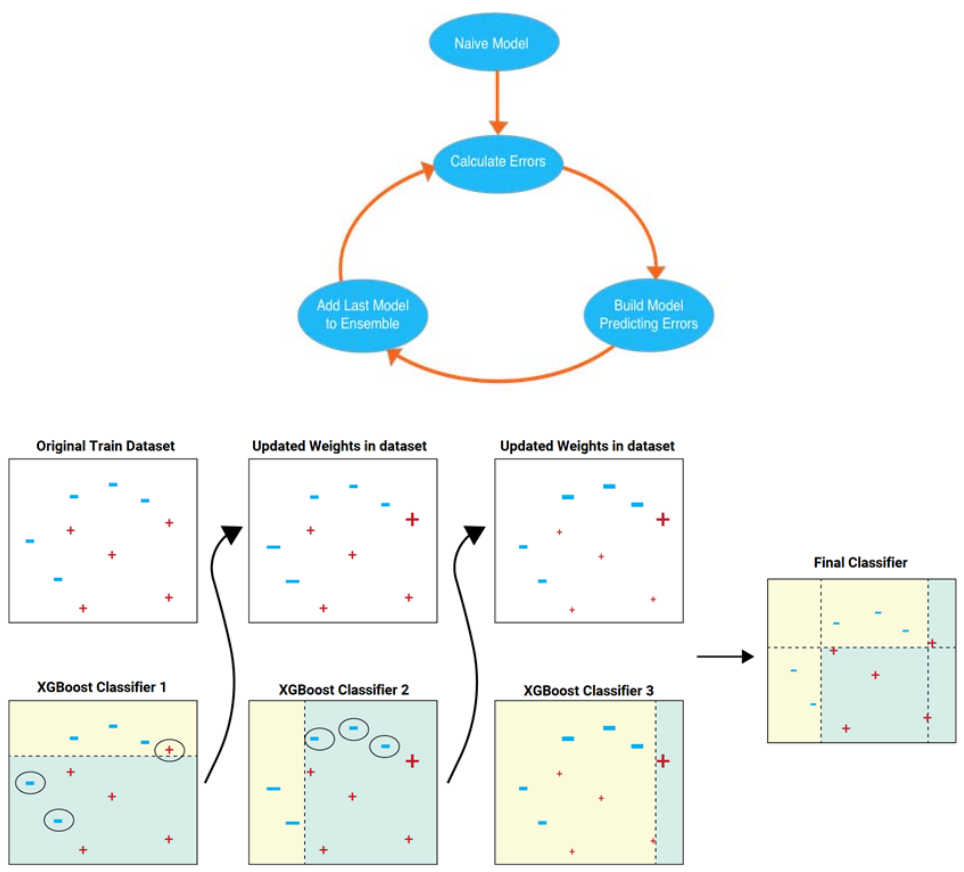

CONCLUSIONS: We conclude that FEs of glycated amino acids are novel risk predictors of EDRF, likely linked to reporting of early-decline of cationic amino acid transporter function in the renal tubular epithelium. Genetic polymorphism of these amino acid transporters has been linked to rapid decline in renal function in genome-wide association studies. Measurement of only 3 glycated amino acids, CMA G-H1 and CML, produced an algorithm with optimal risk prediction of EDRF.

With further validation, including in patients with T2DM and with chronic kidney disease without diabetes, this method may markedly improve clinical risk prediction of EDRF. A patent application has been filed by QU and QF to protect algorithm containing $A 1 C, \log [A C R], F_{C M A}, F_{G-H 1}$, and $[C M L]_{\text {Plasma }}$ features

Acknowledgments: We thank Qatar University and The Qatar Foundation for funding for our research.

Contacts: n.rabbani@qu.edu.qa \& pthornalley@hbku.edu.qa 